\title{
EDITORIAL
}

\section{Culturing expressed breast milk: a costly and (mostly) useless procedure}

\section{Journal of Perinatology (2011) 31, 301; doi:10.1038/jp.2011.21}

There is little doubt that the preferred source of enteral nutrition for preterm infants in the newborn intensive care unit (NICU) is mother's own milk. Multiple clinical studies document important reductions in nosocomial sepsis and necrotizing enterocolitis in preterm infants receiving their mothers' milk compared with those receiving preterm formula. ${ }^{1}$ Longer-term studies suggest improved neurodevelopmental outcomes in extremely low birth weight infants receiving a significant proportion of enteral feeding as mother's milk. ${ }^{2}$ These benefits of human milk have encouraged a resurgence of interest in providing donor human milk to this population of infants when mother's own milk is unavailable. Current evidence suggests that despite the loss/reduction of important immune properties following the pasteurization process, donor milk also protects preterm infants from necrotizing enterocolitis but not from late-onset sepsis. ${ }^{3}$

Accordingly, supporting mothers in providing milk for their very low birth weight infants is an important goal for NICUs. This includes having staff who are skilled and knowledgeable in lactation, as well as processes to assist mothers to safely and effectively pump, collect and store breast milk.

In this issue, Schanler $e t a l^{4}$ provide evidence that even in a NICU with clinical and research expertise in human milk, virtually all pumped breast milk samples (96\%) had bacterial contamination. Most common were samples with low colony counts of Gram-positive organisms or mixed cultures. Patterns of bacterial isolates from the same woman varied over time. Although the organisms cultured from breast milk included most of the pathogens responsible for late-onset sepsis, the predictive value of these cultures for ultimate late-onset sepsis with the same organism was very low. Thus, despite being a non-sterile product, it is likely that the immunological properties of unpasteurized human milk prevent bacterial translocation and invasion.

This study confirms that routine surveillance cultures of pumped and stored human milk in the NICU are not indicated. Beyond the associated costs and the difficulties in interpreting the culture results, such activity may have a negative effect on the willingness of mothers to devote the effort required. Furthermore, such practice by its nature implies a risk to the infant when, in fact, infectious risks in such infants receiving their mothers' milk are reduced.

How should these results be interpreted in light of several reports suggesting an association between breast milk and neonatal sepsis ${ }^{5,6}$ Most such reports are single or small case series documenting an infant(s) with late-onset sepsis and the isolation of the same organism from the infant's mother's breastmilk. Common themes in these cases that may alert clinicians to this problem include recurrent episodes of sepsis with the same organism in a single patient, occurrence of sepsis with the same organism in siblings (twins, triplets), or late-onset sepsis and clinical symptoms of mastitis in the mother. In these situations it may be reasonable to culture mother's milk and arrange treatment for her to enable breastfeeding to continue. It is also important to acknowledge that in these cases the direction of transmission is not clear, particularly if the mother has been doing skin-to-skin care or the infant has been latching at the breast.

An important quality and family-centered goal of any NICU should be to maximize the availability of mother's own milk for its patients. Routine surveillance culture of expressed milk is costly, of little clinical value and may be detrimental to this goal.

\section{Conflict of interest}

The author declares no conflict of interest.

VC Herson

Division of Neonatology, Department of Pediatrics, Connecticut Children's Medical Center, University of Connecticut School of Medicine, Hartford, CT, USA E-mail:Vherson@ccmckids.org

\section{References}

1 Morales Y, Schanler R. Human milk and clinical outcome in VLBW infants: how compelling is the evidence of benefit? Semin Perinatol 2007; 31: 83-88.

2 Vohr BR, Poindexter BB, Dusick AM, McKinly LT, Wright LL, Langer JC et al. Beneficial effects of breast milk in the neonatal intensive care unit on the developmental outcome of extremely low birth weight infants at 18 months of age. Pediatrics 2006; 118: e115-e123.

3 Quigley M, Henderson G, Anthony MY, McGuire W. Formula milk versus donor breast milk for feeding preterm or low birth weight infants. Cochrane Database Syst Rev 2007 Issue 4. Art. No.: CD002971. doi:10.1002/14651858.CD002971.pub2.

4 Schanler R, Fraley J, Lau C, Hurst N, Horvath L. Breastmilk culture and infection in extremely premature infants. J Perinatol 2011; 31: 335-338.

5 Byrne P, Miller C, Justus K. Neonatal group B streptococcal infection related to breastmilk. Breastfeeding Med 2006; 1: 263-270.

6 Godambe S, Shah P, Shah V. Breast milk as a source of late onset neonatal sepsis. Pediatr Infect Dis 2005; 24: 381-382. 